\title{
PHYSIOLOGICAL STUDIES ON THE EFFECT OF FOUR ROOTSTOCKS ON HERNANDINA CLEMENTINE FRUITS II- FRUIT STORABILITY
}

\author{
MOHAMED, M. A. A. ${ }^{1}$ AND M. A. BASSAL ${ }^{2}$
}

1. Fruit Handling Department, Hort. Res. Inst., ARC, Giza,

2. Horticulture Department, Fac. of Agriculture, Suez Canal Univ., Ismailia

(Manuscript received 23 March 2008)

\section{Abstract}

This study was carried out on Hernandina fruits were taken from a private farm at Wady El-Mullak region, Ismailia Governorate during two successive seasons (2005:2006 \& 2006:2007) to determine the effect of four citrus rootstocks on fruit storability at two different low storage temperatures.

Hernandina trees grafted on Sour orange, Carrizo citrange, Cleopatra mandarin and Citrumelo rootstocks were selected for this study. Mature fruits of each treatment in both seasons, were picked, washed, dried, sorted to obtain uniform samples then stored at $5^{\circ} \mathrm{C}$ or $10^{\circ} \mathrm{C}$ and $90-95 \% \mathrm{RH}$ up to 90 and 75 days during the first and the second season, respectively. Physical and chemical properties at 15 day intervals were determined during storage.

Weight loss percentage, decay percentage, total soluble solids and TSS/total acidity ratio increased gradually with the prolonged of storage. In the contrary, fruit gravity, fruit firmness, juice percentage, total acidity and ascorbic acid contents decreased gradually with the increasing of the storage period. Furthermore, fruit color changed directly from yellow to orangeyellow to orange to reddish-orange during storage.

Fruits were produced on Sour orange rootstock showed weight loss incidence, TSS and TSS/total acidity ratio significantly less and decay incidence, fruit gravity, fruit firmness and total acidity were significantly higher than fruits were bearing on the other rootstocks. Rootstock type had no obvious effect on changing of fruit color, fruit juice and ascorbic acid contents changes in stored fruits. However some of these differences return to pre-harvest factors.

Fruits stored at $5^{\circ} \mathrm{C}$ significantly had less weight loss and decay percentage than that fruits stored at $10^{\circ} \mathrm{c}$. Furthermore, storage at $5^{\circ} \mathrm{C}$ significantly reduced the deterioration rate of fruit gravity, fruit coloration, softening, juice content and changes of TSS, total acidity, TSS/total acidity ratio and ascorbic acid in comparison with storage of fruits at $10^{\circ} \mathrm{c}$.

It can be concluded that, Clementine Hernandina fruits are able to be store for 60 days at $5^{\circ} \mathrm{C}$ and $90-95 \% \mathrm{RH}$ with negligible changes of fruit characteristics and quality. Moreover, rootstock types under this study nearly had a little effect on fruit storability. 
1508 PHYSIOLOGICAL STUDIES ON THE EFFECT OF FOUR ROOTSTOCKS ON HERNANDINA CLEMENTINE FRUITS II- FRUIT STORABILTYY

\section{INTRODUCTION}

Citrus is one of the most important fruit crop in Egypt. The total planted area is estimated to be 382027 Faddan in 2006 season produced abut 3211709 tons of fruits in the same year. While the exported quantity in 2005 reached 530909 tons from all citrus species. (General Horticulture Administration, MOA, 2005)

There is a good opportunity for other citrus species than orange to increase its exported quantity to Europe countries under the Egyptian-Europe Association Agreement. The production of the Egyptian local mandarin fruit during a short period and its sensitivity to post harvest and handling management obligate to search about new cultivars, especially early and late ones and has a good ability for marketing and handling processes.

There are new mandarin cultivars and hybrids that have been entered to the Egyptian citrus industry such as Marisol, Clemenules, Hernandina, Nova mandarin, Ellendale tangor and others. These cultivars have been spread in the private sector orchards. However, there is a lack of information about these new cultivars behavior under the Egyptian local conditions, concerning production maturation and post harvest management.

Post-harvest life of mandarin fruits depends on complicated interactions between the physiology of the fruit and its pathogens. Storage temperature is the prime importance. The same variety grown under different climatic conditions may need different storage temperature requirements.

DHallewin et al. (1994) mentioned that storage behavior of Avana mandarin was more affected by rootstock. Also, fruits from trees on Sour orange had the lowest loss from disease incidence during storage, but chilling injury was greatest in fruits produced on Sour orange rootstock. Moreover, quality parameters of fruits were affected by rootstock at harvest and these differences remained during storage and shelf life. The same trend was found by Reynaldo (1999) on Ruby Red grapefruit.

Farih et al. (1995) reported that Clementine cultivars Sidi Aissa, Ain Taoujdate and Cadoux fruits from trees on Cleopatra mandarin conferred greater resistance to storage rots than the other rootstocks (Sour orange, Rough lemon, Troyer citrange). The same trend was reported by Ritenour et al. (2004) on Navel oranges, 'Ray Ruby' grapefruit and 'Oroblanco' fruit. On contrast, Valbuena (1996) indicated that Persian lime fruits on trees grafted on Cleopatra mandarin rootstock had more post-harvest damage and disease incidence significantly higher than that of fruits from trees grown on Volkameriana lemon.

It has been reported that Fremont tangerine fruit on Volkameriana followed by fruits on Rangpur lime had the lowest weight loss percentage during storage while the 
highest were found in fruits on Sour orange rootstocks. Also, the lowest values of juice were found in fruits on Sour orange. Fruits on Carrizo citrange had the highest TSS. Acidity percentage decreased during storage and fruits from trees on Volkameriana rootstocks had the lowest acidity percentage, while that from trees on Sour orange had the highest acidity content (Ali, 2002).

El-Hilali et al. (2003) reported that the highest juice content after 30-days storage was obtained from fruits harvested on C. volkameriana and 'Troyer' citrange and the lowest in fruits from Afourer on 'Sacatan' citrumelo. Fruits from 'Troyer' citrange and 'Sacatan' citrumelo had higher acid content than fruits picked from other rootstocks. In general, total soluble solids in juice were high in fruits from all combinations, except for fruits harvested from Sour orange stored at 4 degrees $\mathrm{C}$.

Schirra, et al. (1997) on Tarocco oranges, reported that total soluble solids content of orange juice increased during storage, while fruit juice content, titratable acidity and ascorbic acid contents decreased. The same results were mentioned by Mohamed et al. (2002 and 2003).

The purpose of this study is to determine the effect of four rootstock types (Sour orange, Carrizo citrange, Cleopatra mandarin and Citrumelo) on Hernandina fruits quality and storability at two different low storage temperatures $\left(5^{\circ} \mathrm{C}\right.$ and $\left.10^{\circ} \mathrm{C}\right)$.

\section{MATERIALS AND METHODS}

This investigation was carried out during two successive seasons (2005:2006 \& 2006:2007) at Hort. Res. Institute, Fruit Handling Department, Giza, Egypt.

Trees were grown in a private farm at "Wady El-Mullak" region, Ismailia Governorate. During February 2005, 36 Hernandina trees grafted on Sour orange, Carrizo citrange, Cleopatra mandarin and "Swingle" citrumelo rootstocks were selected for this study, ( 9 trees for each rootstock). Trees were 7 years oid, healthy, uniformed vigor growth, planted in sandy soil at $2 \times 5 \mathrm{~m}$ under drip irrigation system and subjected to all agriculture practices as ministry agriculture recommendations.

Mature fruit of each treatment in both seasons (the first part of this work), were picked, washed, dried, sorted to obtain uniform samples then stored at $5^{\circ} \mathrm{C}$ or $10^{\circ} \mathrm{C}$ and $90-95 \% \mathrm{RH}$ up to 90 and 75 days during the first and the second season, respectively. Each treatment had six carton boxes (each box had 12 fruits), representing six replicates, three replicates were used for the determination of the physical and chemical properties at 15 dáy intervals. The other three replicates were used to estimate the weight loss and decay percentage (either pathology or physiology) of fruits during storage.

The determination procedures were as follow: 
1- Weight loss percentage: thirty six fruits (three boxes each had twelve fruits) were individually weighted and the differences between its weight at the beginning of the experiment and at the examination day were represented as weight loss percentage.

2- Decay percentage : the weight of unmarketable fruits due to pathological and physiological disorders was determined and this value was calculated.

3- Fruit gravity by dividing fruits size on fruit weight.

4- Fruit firmness $\left(\mathbf{g} / \mathrm{cm}^{2}\right)$ was measured in 6 fruits ( 3 readings per each fruit) by Lfra texture analyzer instrument using a penetrating cylinder of $1 \mathrm{~mm}$ in diameter to a constant distance $5 \mathrm{~mm}$ inside the skin of fruits and by a constant speed $2 \mathrm{~mm}$ per sec. and the peak of resistance was recorded per gram on squire centimeter.

5- Peel colour changes during storage was estimated by a Hunter colorimeter type (Dp-9000) for the estimation of "L", "a" and "b" values and to evaluate colour values as Hue angle and values were calculated according to Mc Guire (1992).

6- Juice content was estimated by squeezing 12 fruits (as three replicates) by handy squeezer and then juice percentage was calculated $(w / w)$.

7- Percentages of total soluble solids (T.S.S. \%), total acidity percentage (as citric acid) and ascorbic acid were estimated according to A.O.A.C. (1985).

Data for all fruit parameters were analyzed as a complete randomized design with factorial treatments as described by Snedecor and Cochran (1980).

\section{RESULTS AND DISCUSSION}

\section{A- Physical properties}

\section{1- Weight loss percentage}

According to data presented in Table (1) weight loss percentage of Hernandina Clementine fruits increased gradually and significantly with the increasing of storage period. Weight loss percentage of fruits stored at $10^{\circ} \mathrm{C}$ was significantly higher than that fruits stored at $5^{\circ} \mathrm{c}$. On the other hand, fruits bearing on Sour orange rootstock had weight loss incidence significantly less than fruits produced on Carrizo and Citrumelo rootstocks. While, weight loss percentage of fruits bearing on Cleopatra rootstock was not different from that found on Sour orange.

These results agree with those mentioned by D'Hallewin et al (1994), Valbuena (1996), Reynaldo (1999) and Ali (2002) who reported that weight loss percentage of citrus fruit was affected by rootstock types.

\section{2- Decay percentage}

Data shown in Table (2) illustrated that decay percentage of fruits increased gradually and significantly with the increasing of storage period. Moreover, fruits 
stored at $10^{\circ} \mathrm{C}$ significantly had decay incidence higher than, that stored at $5^{\circ} \mathrm{C}$ regardless of rootstocks types.

Table 1. Effect of four rootstocks and cold storage on weight loss percentage during storage of "Hernandina" Clementine fruits in 2005/2006 and 2006/2007 seasons.

\begin{tabular}{|c|c|c|c|c|c|c|c|c|c|c|c|c|}
\hline \multicolumn{13}{|c|}{ First season (2005) } \\
\hline St. Temp. & \multicolumn{6}{|c|}{$10^{\circ} \mathrm{C}$} & \multicolumn{5}{|c|}{$5^{\circ} \mathrm{C}$} & \multirow{3}{*}{ Means } \\
\hline \multirow{2}{*}{$\begin{array}{c}\text { St. period } \\
\text { (days) }\end{array}$} & \multicolumn{6}{|c|}{ Rootstock types } & \multicolumn{5}{|c|}{ Rootstock types } & \\
\hline & 1 & 2 & 3 & 4 & \multicolumn{2}{|c|}{ Means } & 1 & 2 & 3 & 4 & Means & \\
\hline 0 & 0.00 & 0.00 & 0.00 & 0.00 & \multicolumn{2}{|c|}{0.00} & 0.00 & 0.00 & 0.00 & 0.00 & 0.00 & 0.00 \\
\hline 15 & 2.62 & 2.13 & 2.35 & 2.47 & \multicolumn{2}{|c|}{2.39} & 1.38 & 1.79 & 1.54 & 1.67 & 1.59 & 1.99 \\
\hline 30 & 4.32 & 4.28 & 4.63 & 3.77 & \multicolumn{2}{|c|}{4.25} & 3.91 & 4.02 & 3.41 & 4.05 & 3.85 & 4.05 \\
\hline 45 & 6.04 & 6.27 & 6.28 & 5.54 & \multicolumn{2}{|c|}{6.04} & 4.74 & 5.02 & 4.91 & 5.24 & 4.98 & 5.51 \\
\hline 60 & 8.13 & 8.97 & 8.46 & 8.85 & \multicolumn{2}{|c|}{8.60} & 6.96 & 7.17 & 6.81 & 7.32 & 7.06 & 7.83 \\
\hline 75 & 10.74 & 11.51 & 10.80 & 11.74 & \multicolumn{2}{|c|}{11.20} & 7.70 & 8.23 & 8.23 & $8: 64$ & 8.20 & 9.70 \\
\hline 90 & 12.62 & 13.21 & 12.89 & 13.36 & \multicolumn{2}{|c|}{13.02} & 9.06 & 9.47 & 8.68 & 9.16 & 9.09 & 11.06 \\
\hline Means & 6.35 & 6.62 & 6.49 & 6.53 & \multicolumn{2}{|c|}{6.50} & 4.82 & 5.10 & 4.79 & $5.15 \cdot$ & 4.97 & \\
\hline \multicolumn{13}{|c|}{ Second season (2006) } \\
\hline 0 & 0.0 & 0.0 & 0.0 & 0.0 & 0.0 & \multicolumn{2}{|c|}{0.0} & 0.0 & 0.0 & 0.0 & 0.0 & 0.0 \\
\hline 15 & 1.9 & 2.5 & 2.0 & 2.3 & 2.2 & \multicolumn{2}{|c|}{1.2} & 1.3 & 1.2 & 1.6 & 1.3 & 1.7 \\
\hline 30 & 3.7 & 4.9 & 3.9 & 4.6 & 4.3 & \multicolumn{2}{|c|}{2.2} & 2.5 & 2.4 & 3.2 & 2.6 & 3.4 \\
\hline 45 & 6.9 & 7.9 & 6.7 & 7.9 & 7.4 & \multicolumn{2}{|c|}{4.6} & 4.7 & 4.6 & 5.6 & 4.9 & 6.1 \\
\hline 60 & 9.0 & 10.4 & 8.9 & 10.1 & 9.6 & \multicolumn{2}{|c|}{6.1} & 7.0 & 5.9 & 7.7 & 6.7 & 8.1 \\
\hline 75 & 11.1 & 13.0 & 11.6 & 12.3 & 12.0 & \multicolumn{2}{|c|}{7.7} & 9.3 & 7.3 & 9.8 & 8.5 & 10.2 \\
\hline Means & 5.4 & 6.5 & 5.5 & 6.2 & 5.9 & 3. & & 4.1 & 3.6 & 4.6 & 4.0 & \\
\hline \multicolumn{6}{|c|}{ Rootstock means } & \multicolumn{6}{|c|}{ Abbreviations: } & \\
\hline \multicolumn{2}{|c|}{ Rootstock } & 1 & 2 & 3 & 4 & \multicolumn{4}{|c|}{$1=$ Sour orange } & \multicolumn{3}{|c|}{$2=$ Carrizo } \\
\hline \multicolumn{2}{|c|}{$1^{\text {st }}$ season } & 5.6 & 5.9 & 5.6 & 5.8 & $3=c$ & opatra & & & $4=\mathrm{Cit}$ & imelo & \\
\hline $2^{\text {nt }}$ seaso & & 4.5 & 5.3 & 4.5 & 5.4 & $A=S$ & rage $\mathrm{Te}$ & & & $B=$ Rootstoc & types & \\
\hline & S.D. at & $5 \%$ & & & & $C=S$ & rage pe & iod in $\mathrm{d}$ & & & & \\
\hline Factors & & $A$ & & B & & $\mathrm{C}$ & $a^{\prime}$ & & $a^{*} c$ & $b^{*}$ & & $a * b * c$ \\
\hline $1^{\text {st }}$ seasc & & 0.16 & & 0.18 & & 0.24 & & & 0.34 & N.S & & N.S. \\
\hline $2^{\text {nd }}$ seasc & & 0.16 & & 0.22 & & 0.27 & & & 0.39 & 0.5 & & N.S. \\
\hline
\end{tabular}

On the other hand, data cleared that Hernandina fruits produced on Sour orange rootstock had significantly higher decay incidence than that fruits on other rootstocks. Furthermore, there were significant differences among fruits bearing on Carrizo, Cleopatra and Citrumelo concerning decay incidence during the two seasons in this investigation. 
1512 PHYSIOLOGICAL STUDIES ON THE EFFECT OF FOUR ROOTSTOCKS ON HERNANDINA CLEMENTINE FRUITS II- FRUIT STORABILITY

These results are in agreement with those obtained by El-Zeftawi et al. (1989), Valbuena (1996) and Ritenour et al. (2004). They demonstrated that decay incidence of citrus fruit was significantly different according to rootstock types.

Table 2. Effect of four rootstocks and cold storage on decay percentage during storage of "Hernandina" Clementine fruits in 2005/2006 and 2006/2007 seasons.

\begin{tabular}{|c|c|c|c|c|c|c|c|c|c|c|c|}
\hline \multicolumn{12}{|c|}{ First season (2005) } \\
\hline $\begin{array}{c}\text { St. } \\
\text { Temp. }\end{array}$ & \multicolumn{5}{|c|}{$10^{\circ} \mathrm{C}$} & \multicolumn{5}{|c|}{$5^{\circ} \mathrm{C}$} & \multirow{3}{*}{ Means } \\
\hline \multirow{2}{*}{$\begin{array}{c}\text { St. } \\
\text { period } \\
\text { (days) }\end{array}$} & \multicolumn{5}{|c|}{ Rootstock types } & \multicolumn{5}{|c|}{ Rootstock types } & \\
\hline & 1 & 2 & 3 & 4 & Means & 1 & 2 & 3 & 4 & Means & \\
\hline 0 & 0.00 & 0.00 & 0.00 & 0.00 & 0.00 & 0.00 & 0.00 & 0.00 & 0.00 & 0.00 & 0.00 \\
\hline 15 & 0.00 & 0.00 & 0.00 & 0.00 & 0.00 & 0.00 & 0.00 & 0.00 & 0.00 & 0.00 & 0.00 \\
\hline 30 & 23.25 & 6.05 & 6.67 & 9.67 & 11.41 & 5.88 & 7.17 & 7.14 & 6.61 & 6.70 & 9.05 \\
\hline 45 & 26.76 & 6.05 & 9.98 & 14.11 & 14.23 & 0.17 & 14.34 & 10.48 & 9.81 & 8.70 & 11.46 \\
\hline 60 & 39.52 & 26.63 & 23.08 & 27.19 & 29.10 & 24.49 & 15.29 & 13.5 & 9.54 & 15.73 & 22.42 \\
\hline 75 & 39.52 & 33.29 & 26.83 & 37.20 & 34.21 & 28.60 & 9.68 & 13.5 & 13.01 & 16.22 & 25.21 \\
\hline 90 & 62.70 & 46.57 & 47.47 & 49.52 & 51.56 & 32.90 & 13.51 & 13.5 & 19.30 & 19.83 & 35.69 \\
\hline Means & 27.39 & 16.94 & 16.29 & 19.67 & 20.07 & 13.15 & 8.57 & 8.34 & 8.32 & 9.60 & \\
\hline \multicolumn{11}{|c|}{ Second season (2006) } & • \\
\hline 0 & 0.0 & 0.0 & 0.0 & 0.0 & .0 .00 & 0.0 & 0.0 & 0.0 & 0.0 & 0.00 & 0.00 \\
\hline 15 & 3.1 & 0.0 & 3.3 & 6.0 & 3.10 & 0.0 & 0.0 & 0.0 & 0.0 & 0.00 & 1.55 \\
\hline 30 & 9.5 & 6.8 & 10.4 & 6.0 & 8.17 & 0.0 & 0.0 & 0.0 & 0.0 & 0.00 & 4.08 \\
\hline 45 & 32.1 & 12.9 & 10.4 & 15.7 & 17.78 & 10.4 & 0.0 & 3.0 & 0.0 & 3.35 & 10.56 \\
\hline 60 & 32.1 & 12.9 & 17.0 & 15.7 & 19.41 & 17.0 & 3.5 & 10.3 & 3.2 & 8.48 & 13.94 \\
\hline 75 & 32.1 & 16.1 & 23.3 & 15.7 & 21.78 & 27.3 & 9.5 & 10.3 & 10.2 & 14.29 & 18.04 \\
\hline Means & 18.15 & 8.12 & 10.72 & 9.83 & 11.71 & 9.10 & 2.15 & 3.93 & 2.23 & 4.35 & \\
\hline \multicolumn{6}{|c|}{ Rootstock means } & \multicolumn{6}{|c|}{ Abbreviations: } \\
\hline \multicolumn{2}{|c|}{ Rootstock } & 1 & 2 & 3 & 4 & \multicolumn{4}{|c|}{$1=$ Sour orange } & \multicolumn{2}{|c|}{$2=$ Carrizo } \\
\hline \multicolumn{2}{|c|}{$1^{\text {st }}$ season } & 20.3 & 12.8 & 12.3 & 14.0 & \multicolumn{4}{|c|}{$3=$ Cleopatra } & \multicolumn{2}{|c|}{$4=$ Citrumelo } \\
\hline $2^{\text {nd }} \mathrm{se}$ & eason & 13.6 & 5.1 & 7.3 & 6.0 & & $=$ Stora & Temp. & & B= Rootst & ck types \\
\hline & L.S.D. at & $5 \%$ & 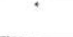 & & & & & $\mathrm{C}=\mathrm{S}$ & rage perio & in days & \\
\hline Fact & tors & A & & B & & & C & $a * b$ & . $a^{*} c$ & $\mathrm{~b} * \mathrm{c}$ & $a * b * c$ \\
\hline $1^{\text {st }} \mathrm{se}$ & eason & 3.17 & & 4.48 & & & 5.93 & N.S. & 8.39 & N.S. & N.S. \\
\hline $2^{\text {nd }} \mathrm{se}$ & eason & 2.38 & & 3.36 & & & 12 & N.S. & 5.82 & 8.24 & N.S. \\
\hline
\end{tabular}




\section{3- Fruit gravity $\left(\mathrm{g} / \mathrm{cm}^{2}\right)$}

Data shown in Table (3) demonstrated that fruit gravity decreased gradually and significantly with the increasing of storage period during the two seasons of this investigation. Data also indicated that storage of fruits at $5{ }^{\circ} \mathrm{C}$ significantly reduced the deterioration rate of fruit gravity during storage in comparison with those stored at $10^{\circ} \mathrm{C}$. On the other hand, gravity of fruits bearing on Sour orange was significantly higher in comparison with those on the other rootstocks. However, these differences return to the effect of rootstock during pre-harvest period and still appear during storage period.

Table 3. Effect of four rootstocks and cold storage on fruits gravity during storage of "Hernandina" Clementine fruits in 2005/2006 and 2006/2007 seasons.

\begin{tabular}{|c|c|c|c|c|c|c|c|c|c|c|}
\hline \multicolumn{11}{|c|}{ First season (2005) } \\
\hline St. Temp. & \multicolumn{4}{|c|}{$10^{\circ} \mathrm{C}$} & \multicolumn{5}{|c|}{$5^{\circ} \mathrm{C}$} & \multirow{3}{*}{ Means } \\
\hline \multirow{2}{*}{$\begin{array}{c}\text { St. period } \\
\text { (days) }\end{array}$} & \multicolumn{4}{|c|}{ Rootstock types } & \multicolumn{5}{|c|}{ Rootstock types } & \\
\hline & 1 & \begin{tabular}{l|l}
2 & 3 \\
\end{tabular} & \begin{tabular}{l|l}
3 & 4 \\
\end{tabular} & 4 Means & 1 & 2 & 3 & 4 & Means & \\
\hline 0 & \begin{tabular}{|l|l|}
0.97 & 0. \\
\end{tabular} & \begin{tabular}{l|l}
.93 & 0.9 \\
\end{tabular} & .950. & \begin{tabular}{l|l|}
93 & 0.94 \\
9
\end{tabular} & 0.97 & 0.93 & 0.95 & 0.93 & 0.94 & 0.94 \\
\hline 15 & \begin{tabular}{|l|l|l}
0.94 & 0. \\
\end{tabular} & \begin{tabular}{l|l}
0.90 & 0.9 \\
\end{tabular} & \begin{tabular}{l|l}
.92 & 0. \\
\end{tabular} & \begin{tabular}{l|l}
92 & 0.92 \\
\end{tabular} & 0.96 & 0.92 & 0.94 & 0.92 & 0.93 & 0.93 \\
\hline 30 & \begin{tabular}{|l|l}
0.91 & 0. \\
\end{tabular} & $. 9 0 \longdiv { 0 . 9 }$ & \begin{tabular}{l|l}
92 & 0. \\
\end{tabular} & \begin{tabular}{l|l|}
90 & 0.90 \\
\end{tabular} & 0.94 & 0.92 & 0.92 & 0.89 & 0.92 & 0.91 \\
\hline 45 & \begin{tabular}{|l|l|}
0.87 & 0. \\
\end{tabular} & \begin{tabular}{l|l}
.87 & 0.7 \\
\end{tabular} & \begin{tabular}{l|l}
.75 & 0.8 \\
\end{tabular} & \begin{tabular}{l|l|}
87 & 0.84 \\
\end{tabular} & 0.91 & 0.90 & 0.89 & 0.89 & 0.90 & 0.87 \\
\hline 60 & \begin{tabular}{|l|l}
0.80 & 0. \\
\end{tabular} & \begin{tabular}{l|l}
.72 & 0.7 \\
\end{tabular} & \begin{tabular}{l|l}
.74 & 0.8 \\
\end{tabular} & \begin{tabular}{l|l}
80 & 0.76 \\
\end{tabular} & 0.87 & 0.74 & 0.80 & 0.83 & 0.81 & 0.79 \\
\hline 75 & $\begin{array}{ll}0.78 & 0 . \\
\end{array}$ & \begin{tabular}{l|l}
.73 & 0.7 \\
\end{tabular} & \begin{tabular}{l|l}
.72 & 0. \\
\end{tabular} & \begin{tabular}{l|l|}
75 & 0.75 \\
\end{tabular} & 0.85 & 0.73 & 0.79 & 0.82 & 0.80 & 0.77 \\
\hline 90 & \begin{tabular}{|l|l|l}
0.74 & 0. \\
\end{tabular} & \begin{tabular}{l|l}
.64 & 0.6 \\
\end{tabular} & \begin{tabular}{l|l}
61 & 0. \\
\end{tabular} & \begin{tabular}{l|l}
67 & 0.67 \\
\end{tabular} & 0.81 & 0.72 & 0.72 & 0.76 & 0.75 & 0.71 \\
\hline Means $] 0$ & $0.86] 0$. & $.81[0.8$ & $80 \mid 0.8$ & \begin{tabular}{l|l}
83 & 0.83 \\
\end{tabular} & 0.90 & 0.84 & 0.86 & 0.86 & 0.86 & \\
\hline \multicolumn{11}{|c|}{ Second season (2006) } \\
\hline 0 & 0.910. & $\begin{array}{l}0.84 \\
0.8\end{array}$ & $\begin{array}{ll}.86 & 0 . \\
\end{array}$ & \begin{tabular}{l|l}
84 & 0.86 \\
\end{tabular} & 0.91 & 0.84 & 0.86 & 0.84 & 0.86 & 0.86 \\
\hline 15 & \begin{tabular}{|l|l|l|}
0.81 & 0. \\
\end{tabular} & \begin{tabular}{l|l}
.73 & 0.6 \\
\end{tabular} & \begin{tabular}{l|l}
.69 & 0. \\
\end{tabular} & \begin{tabular}{l|l|}
75 & 0.74 \\
\end{tabular} & 0.85 & 0.75 & 0.73 & 0.76 & 0.77 & 0.76 \\
\hline 30 & 0.780. & .710 .6 & 670. & \begin{tabular}{l|l}
76 & 0.73 \\
\end{tabular} & 0.81 & 0.73 & 0.71 & 0.78 & 0.76 & 0.74 \\
\hline 45 & 0.690. & \begin{tabular}{l|l}
.62 & 0.6 \\
\end{tabular} & \begin{tabular}{l|l}
63 & 0. \\
\end{tabular} & \begin{tabular}{l|l}
68 & 0.65 \\
\end{tabular} & 0.82 & 0.72 & 0.64 & 0.77 & 0.74 & 0.70 \\
\hline 60 & 0.650. & $0.66 \mid 0.5$ & \begin{tabular}{l|l}
.54 & 0. \\
\end{tabular} & \begin{tabular}{l|l}
65 & 0.62 \\
\end{tabular} & 0.73 & 0.62 & 0.61 & 0.75 & 0.68 & 0.65 \\
\hline 75 & 0.570. & \begin{tabular}{l|l}
0.60 & 0.6 \\
\end{tabular} & \begin{tabular}{l|l}
66 & 0. \\
\end{tabular} & \begin{tabular}{l|l}
60 & 0.61 \\
\end{tabular} & 0.67 & 0.67 & 0.65 & 0.66 & 0.66 & 0.64 \\
\hline Means $] 0$ & 0.740. & $.69 \mid 0.6$ & 670. & 71) 0.70 & 0.80 & 0.72 & 0.70 & 0.76 & 0.75 & \\
\hline \multicolumn{5}{|c|}{ Rootstock means } & \multicolumn{6}{|c|}{ Abbreviations: } \\
\hline$\frac{\text { Rootstock }}{1^{\text {st }} \text { season }}$ & \begin{tabular}{|c|}
1 \\
0.88
\end{tabular} & 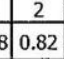 & \begin{tabular}{|c|}
3 \\
2.83
\end{tabular} & \begin{tabular}{|c}
4 \\
0.85
\end{tabular} & \multicolumn{3}{|c|}{$1=$ Sour orange } & \multicolumn{3}{|c|}{$2=$ Carrizo } \\
\hline $2^{\text {nd }}$ season & 0.77 & 70.71 & 0.69 & 0.74 & \multicolumn{3}{|c|}{$3=$ Cleopatra } & \multicolumn{3}{|c|}{$4=$ Citrumelo } \\
\hline \multirow{2}{*}{\multicolumn{5}{|c|}{ L.S.D. at $5 \%$}} & \multicolumn{3}{|c|}{$\mathrm{A}=$ Storage Temp. } & \multicolumn{3}{|c|}{ B=Rootstock types } \\
\hline & & & & & \multicolumn{6}{|c|}{$\mathrm{C}=$ Storage period in days } \\
\hline Factors & & A & $B$ & C & $a * b$ & & & $\mathrm{~b}^{*}$ & & $a * b * c$ \\
\hline $1^{\text {st }}$ season & 0.0 & $017 \quad 0$ & 0.024 & 0.031 & N.S. & & S. & 0.06 & & N.S. \\
\hline $2^{\text {nd }}$ season & \begin{tabular}{l|l}
$n$ & 0.0 \\
\end{tabular} & \begin{tabular}{l|l}
018 & 0 \\
\end{tabular} & 0.026 & $\cdots 0.036$ & N.S. & & I.S. & 0.07 & & N.S. \\
\hline
\end{tabular}

\section{4- Fruit color:}

Fruit color data presented in Table (4) as Hue angel value cleared that, fruit color changed directly from Yellow (Hue angel is more than $60^{\circ}$ ) to orange-yellow to orange to reddish-orange (Hue angel is less than $45^{\circ}$ ) during storage in the two seasons of this study. Moreover, $5^{\circ} \mathrm{C}$ storage temperatures significantly decreased the coloration rate during storage compared with $10^{\circ} \mathrm{C}$ during both seasons. 
Data also indicated that, rootstock type had no effect on color changes in stored fruits during the first season. While in the second one, color changes of fruits bearing on Carrizo and Citrumelo were more accelerated than those on the two other rootstocks. However, these changes were due to the pre-harvest period effect.

Table 4. Effect of four rootstocks and cold storage on fruits color, represented as hue angle values, during storage of "Hernandina" Clementine fruits in 2005/2006 and 2006/2007 seasons.

\begin{tabular}{|c|c|c|c|c|c|c|c|c|c|c|c|}
\hline \multicolumn{12}{|c|}{ First season (2005) } \\
\hline $\begin{array}{l}\text { St. } \\
\text { Temp. }\end{array}$ & \multicolumn{5}{|c|}{$10^{\circ} \mathrm{C}$} & \multicolumn{5}{|c|}{$5^{\circ} \mathrm{C}$} & \multirow{3}{*}{ Means } \\
\hline \multirow{2}{*}{$\begin{array}{c}\text { St. } \\
\text { period } \\
\text { (days) }\end{array}$} & \multicolumn{5}{|c|}{ Rootstock types } & \multicolumn{5}{|c|}{ Rootstock types } & \\
\hline & 1 & 2 & 3 & 4 & $\begin{array}{c}\text { Mea } \\
\text { ns }\end{array}$ & 1 & 2 & 3 & 4 & Means & \\
\hline 0 & 67.7 & 64.9 & 62.3 & 64.1 & 64.7 & 67.7 & 64.9 & 62.3 & 64.1 & 64.74 & 64.7 \\
\hline 15 & 61.4 & 60.1 & 56.1 & 57.7 & 58.8 & 67.8 & 65.0 & 59.4 & 62.5 & 63.67 & 61.2 \\
\hline 30 & 58.8 & 59.4 & 55.9 & 57.4 & 57.9 & 63.3 & 64.0 & 61.0 & 58.8 & 61.79 & 59.8 \\
\hline 45 & 55.1 & 55.3 & 49.9 & 51.3 & 52.9 & 67.9 & 65.1 & 56.5 & 60.9 & 62.59 & 57.7 \\
\hline 60 & 49.8 & 54.0 & 49.4 & 50.7 & 51.0 & 58.9 & 63.2 & 59.7 & 53.5 & 58.83 & 54.9 \\
\hline 75 & 48.1 & 45.0 & 45.9 & 47.1 & 46.5 & 59.1 & 57.2 & 50.7 & 51.5 & 54.63 & 50.8 \\
\hline 90 & 48.7 & 40.5 & 42.9 & 42.3 & 43.6 & 52.4 & 46.2 & 49.4 & 45.7 & 48.40 & 46.0 \\
\hline Means & 55.7 & 54.2 & 51.8 & 53.0 & 53.6 & 62.5 & 60.78 & 57.0 & 56.7 & 59.2 & \\
\hline \multicolumn{12}{|c|}{ Second season (2006) } \\
\hline 0 & 68.6 & 63.0 & 66.1 & 64.7 & 65.6 & 68.6 & 63.0 & 66.1 & 64.7 & 65.6 & 65.6 \\
\hline 15 & 53.2 & 51.4 & 48.8 & 53.4 & 51.7 & 54.8 & 48.1 & 59.0 & 54.5 & 54.1 & 52.9 \\
\hline 30 & 50.2 & 47.0 & 49.8 & 44.6 & 47.9 & 49.9 & 49.2 & 55.6 & 51.4 & 51.5 & 49.7 \\
\hline 45 & 46.9 & 42.0 & 51.8 & 35.6 & 44.1 & 45.0 & 50.6 & 52.6 & 49.2 & 49.3 & 46.7 \\
\hline 60 & 45.6 & 36.8 & 46.9 & 38.6 & 42.0 & 47.1 & 48.8 & 53.5 & 47.5 & 49.2 & 45.6 \\
\hline 75 & 44.3 & 30.5 & 43.0 & 42.1 & 40.0 & 49.3 & 46.9 & 54.5 & 45.6 & 49.1 & 44.5 \\
\hline Means & 51.5 & 45.1 & 51.1 & 46.5 & 48.5 & 52.5 & 51.1 & 56.9 & 52.1 & 53.1 & \\
\hline \multicolumn{6}{|c|}{ Rootstock means } & \multicolumn{6}{|c|}{ Abbreviations: } \\
\hline Rootstock & 1 & 2 & \multicolumn{2}{|l|}{3} & 4 & \multicolumn{4}{|c|}{$1=$ Sour orange } & \multicolumn{2}{|c|}{$2=$ Carrizo } \\
\hline $1^{\text {st }}$ season & 59.1 & 57.5 & 54.4 & & 54.8 & \multicolumn{4}{|c|}{$3=$ Cleopatra } & \multicolumn{2}{|c|}{$4=$ Citrumelo } \\
\hline $2^{\text {nd }}$ season & 52.0 & 48.1 & 54.0 & & 49.3 & \multicolumn{4}{|c|}{$A=$ Storage Temp. } & \multicolumn{2}{|c|}{$\mathrm{B}=$ Rootstock types } \\
\hline \multicolumn{6}{|c|}{ L.S.D. at $5 \%$} & \multicolumn{6}{|c|}{$\mathrm{C}=$ Storage period in days } \\
\hline Factors & A & $B$ & 3 & $\mathrm{C}$ & & $a * b$ & & $a * c$ & & $b^{* c} \mathrm{c}$ & $a * b * c$ \\
\hline $\begin{array}{l}1^{\text {st }} \\
\text { season }\end{array}$ & 1.32 & N. & S. & 2.48 & & N.S. & & 3.5 & & N.S. & N.S. \\
\hline $\begin{array}{l}2^{\text {nd }} \\
\text { season }\end{array}$ & 2.32 & 3.2 & 29 & 4.03 & & N.S. & & N.S. & & N.S. & N.S. \\
\hline
\end{tabular}

These results are in harmony with those obtained by Schirra, et al. (1997) who demonstrated that Star Ruby grapefruit color was nearly not affected by storage period, but storage temperature resulted in increase in $\mathrm{a} / \mathrm{b}$ hunter value ratio at the high storage temperature. 


\section{5- Fruit firmness :( $\left.\mathrm{gm} / \mathrm{cm}^{2}\right)$}

Fruit firmness (Table 5) of Hernandina significantly decreased with the extension of storage period regardless of rootstock or storage temperature. Moreover, $5^{\circ} \mathrm{C}$ storage temperature significantly reduced fruit softening rate during storage regardless of rootstock types in this study.

Table 5. Effect of four rootstocks and cold storage on fruit firmness during storage of "Hernandina" Clementine fruits in 2005/2006 and 2006/2007 seasons.

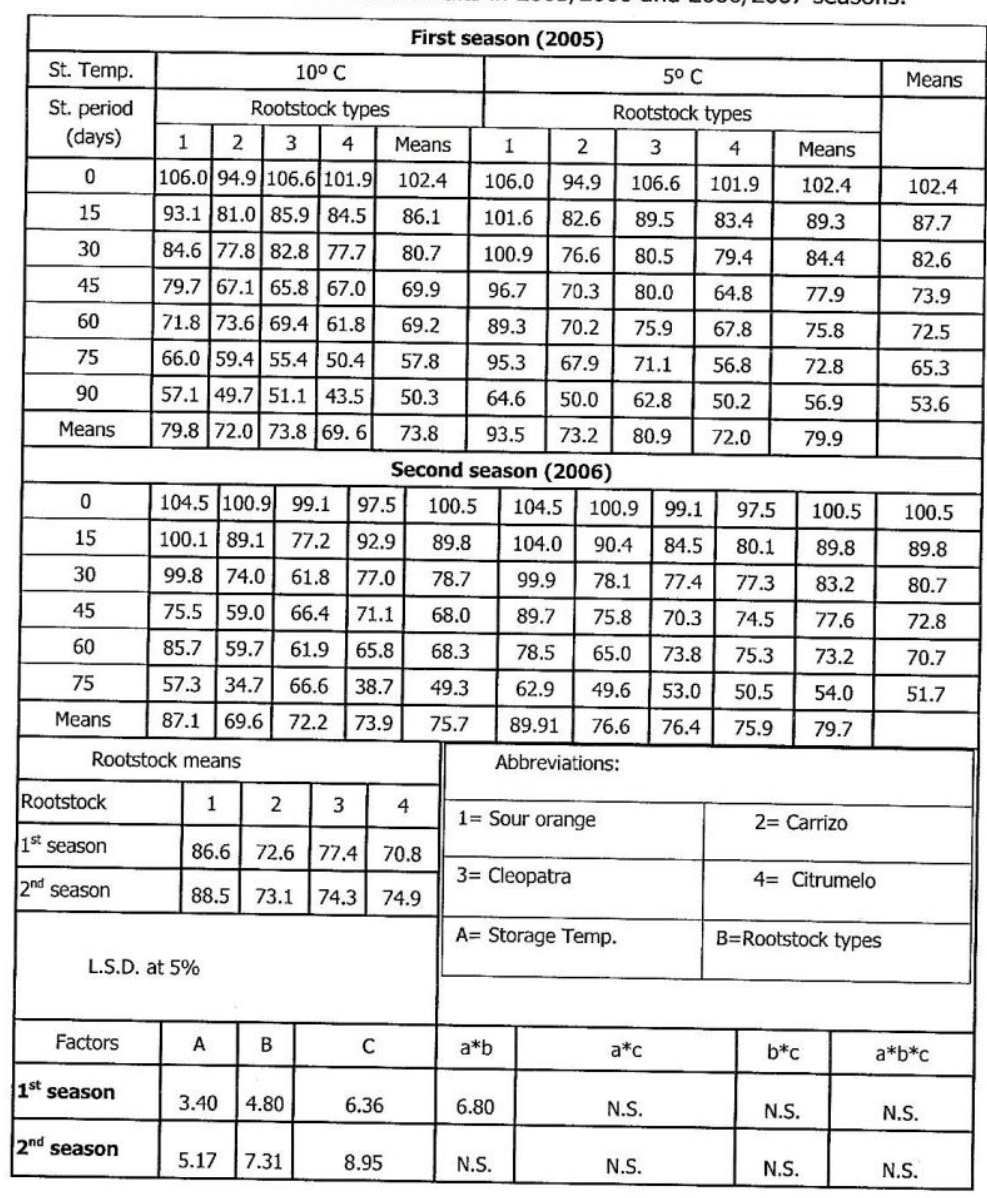

On the other hand, firmness of fruits bearing on Sour orange were significantly higher than those on the other rootstocks during both seasons in this study, however these differences return to pre-harvest factors. 
1516 PHYSIOLOGICAL STUDIES ON THE EFFECT OF FOUR ROOTSTOCKS ON HERNANDINA CLEMENTINE FRUITS II- FRUIT STORABILITY

These results are in line with those demonstrated by Alferez et al. (2005) who mentioned that, orange fruit firmness significantly decreased with the increasing of cold storage period. Furthermore, these results are in harmony with those mentioned by El-Hilali et al. (2003) who mentioned that mandarin fruit firmness during storage significantly differed according to rootstock.

\section{6- Juice percentage:}

Data shown in Table (6) indicated that juice content of fruits decreased gradually and significantly with the increasing of storage period. Moreover, $5^{\circ} \mathrm{C}$ storage temperatures reduced the deterioration rate of juice content compared with $10^{\circ} \mathrm{C}$ storage temperature. Furthermore, data indicated that rootstock types had no effect on fruit content of juice during storage.

These results disagree with those obtained by Ali (2002) and El-Hilali et al. (2003) who mentioned that juice percentage of mandarin stored fruit were difference according to rootstock types. Furthermore, these results are in harmony with those illustrated by Ramanjulu and Reddy (1989), Mohamed et al. (2002) and Mohamed et al. (2003) who demonstrated that juice percentage of Valencia orange and grapefruit fruit decreased with the extension of storage period.

\section{B - Chemical properties:}

1- Total soluble solids, total acidity, TSS/acid ratio and Ascorbic acid contents:

According to data shown in Tables $(7,8,9$ and 10) it is clear that, total soluble solids and TSS/acid ratio increased while total acidity and ascorbic acid contents decreased gradually and significantly with the increasing of storage period.

Data also indicated that $5^{\circ} \mathrm{C}$ storage temperature reduced the changes rate of TSS, total acidity, TSS/acid ratio and ascorbic acid during storage. However, this effect was not significant in TSS contents during the first season and ascorbic acid content during the two seasons of this study.

Data also cleared that fruits bearing on trees grafted on Carrizo and Citrumelo rootstocks had TSS contents higher than those on Sour orange and Cleopatra rootstocks during both seasons. On the other hand, data illustrated that fruits from trees grafted on Carrizo, Cleopatra and Citrumelo rootstocks had total acidity contents less and TSS/acid ratio higher than fruits produced on Sour orange. On contrast, in this respect, ascorbic acid content had no obvious trend during the two seasons. During the first season, fruits on Cleopatra and Sour orange rootstock had the lowest ascorbic acid content, while during the second season this trend was inverted completely. However, it appears that these effects were return to the effect of these rootstocks during pre harvest period. 
Table 6. Effect of four rootstocks and cold storage on juice percentage during storage of "Hernandina" Clementine fruits in 2005/2006 and 2006/2007 seasons.

\begin{tabular}{|c|c|c|c|c|c|c|c|c|c|c|}
\hline \multicolumn{11}{|c|}{ First season (2005) } \\
\hline St. Temp. & \multicolumn{4}{|c|}{$10^{\circ} \mathrm{C}$} & \multicolumn{5}{|c|}{$5^{\circ} \mathrm{C}$} & \multirow{3}{*}{ Means } \\
\hline St. period & \multicolumn{4}{|c|}{ Rootstock types } & \multicolumn{5}{|c|}{ Rootstock types } & \\
\hline (days) & 1 & 3 & 4 & Means & 1 & 2 & 3 & 4 & Means & \\
\hline 0 . & 48.848 & 18.048 .3 & 47.1 & 48.0 & 48.6 & 48.3 & 49.2 & 50.4 & 49.1 & 48.6 \\
\hline 15 & 44.847 & \begin{tabular}{lll|}
7.1 & 47.9 \\
\end{tabular} & 45.0 & 46.2 & 48.7 & 48.9 & 48.9 & 51.7 & 49.6 & 47.9 \\
\hline 30 & 42.244 & 4.644 .1 & 41.5 & 43.1 & 49.5 & 44.5 & 48.4 & 46.0 & 47.1 & 45.1 \\
\hline 45 & 40.846 & 6.247 .5 & 42.9 & 44.3 & 48.9 & 49.5 & 48.6 & 53.0 & 50.0 & 47.2 \\
\hline 60 & 41.341. & 1.239 .9 & 35.8 & 39.6 & 50.4 & 40.8 & 47.6 & 41.6 & 45.1 & 42.3 \\
\hline 75 & 35.641. & 1.341 .3 & 34.9 & 38.3 & 43.9 & 41.5 & 45.7 & 43.0 & 43.5 & 40.9 \\
\hline 90 & 34.436. & 6.633 .4 & 35.0 & 34.9 & 32.3 & 39.7 & 36.1 & 40.5 & 37.2 & 36.0 \\
\hline Means & 41.143. & \begin{tabular}{l|l}
13.6 & 43.2
\end{tabular} & 40.3 & 42.1 & 46.0 & 44.7 & 46.4 & 46.6 & 45.9 & \\
\hline \multicolumn{11}{|c|}{ Second season (2006) } \\
\hline 0 & 50.247 & 7.149 .6 & 49.2 & 49.0 & 50.2 & 47.1 & 49.6 & 49.2 & 49.0 & 49.0 \\
\hline 15 & 49.149 & 19.745 .6 & 48.0 & 48.1 & 45.4 & 42.6 & 47.1 & 44.5 & 44.9 & 46.5 \\
\hline 30 & 46.039 & 39.944 .1 & 42.7 & 43.2 & 44.7 & 40.9 & 44.4 & 44.9 & 43.7 & 43.4 \\
\hline . 45 & 42.930 & $\begin{array}{lll}30.1 & 42.6 \\
\end{array}$ & 37.3 & 38.2 & 44.0 & 39.1 & 41.8 & 42.5 & 41.8 & 40.0 \\
\hline 60 & 37.231 & 1.438 .9 & 34.0 & 35.4 & 39.0 & 38.0 & 37.8 & 40.0 & 38.7 & 37.0 \\
\hline 75 & 31.532 & 2.635 .3 & 30.7 & 32.5 & 34.1 & 34.9 & 33.8 & 34.7 & 34.4 & 33.4 \\
\hline Means & 42.838 & 8.542 .7 & 40.3 & 41.1 & 42.9 & 40.4 & 42.4 & 42.6 & 42.1 & \\
\hline \multicolumn{5}{|c|}{ Rootstock means } & & & & & & \\
\hline Rootstock & 1 & 2 & 3 & 4 & \multicolumn{6}{|c|}{ Abbreviations: } \\
\hline & & & & & \multicolumn{3}{|c|}{$1=$ Sour orange } & \multicolumn{3}{|c|}{$2=$ Carrizo } \\
\hline $1^{\text {st }}$ season & 43.6 & 44.1 & 44.8 & 43.5 & \multicolumn{3}{|c|}{ 3= Cleopatra } & \multicolumn{3}{|c|}{$4=$ Citrumelo } \\
\hline $2^{\text {nd }}$ season & 42.8 & 39.5 & 42.5 & 41.5 & & ge Tem & & \multicolumn{3}{|c|}{$B=$ Rootstock types } \\
\hline \multicolumn{5}{|c|}{ L.S.D. at $5 \%$} & \multicolumn{6}{|c|}{$\mathrm{C}=$ Storage period in days } \\
\hline Factors & A & B & $\mathrm{C}$ & & $a * b$ & \multicolumn{2}{|c|}{$a * c$} & $b^{*} \mathrm{c}$ & \multicolumn{2}{|c|}{$a * b * c$} \\
\hline $1^{\text {st }}$ season & 1.79 & N.S. & 3.58 & \multicolumn{2}{|r|}{ N.S. } & \multicolumn{2}{|c|}{ N.S. } & N.S. & \multicolumn{2}{|r|}{ N.S. } \\
\hline $2^{\text {nd }}$ season & N.S. & N.S. & 3.21 & \multicolumn{2}{|r|}{ N.S. } & \multicolumn{2}{|c|}{ N.S. } & N.S. & \multicolumn{2}{|r|}{ N.S. } \\
\hline
\end{tabular}


1518 PHYSIOLOGICAL STUDIES ON THE EFFECT OF FOUR ROOTSTOCKS ON HERNANDINA CLEMENTINE FRUITS II- FRUIT STORABILITY

Table 7. Effect of four rootstocks and cold storage on TSS percentage during storage of "Hernandina" Clementine fruits in 2005/2006 and 2006/2007 seasons.

\begin{tabular}{|c|c|c|c|c|c|c|c|c|c|c|c|c|}
\hline \multicolumn{13}{|c|}{ First season (2005) } \\
\hline St. Temp. & \multicolumn{6}{|c|}{$10^{\circ} \mathrm{C}$} & \multicolumn{5}{|c|}{$5^{\circ} \mathrm{C}$} & \multirow{3}{*}{ Means } \\
\hline \multirow{2}{*}{$\begin{array}{c}\text { St. period } \\
\text { (days) }\end{array}$} & \multicolumn{6}{|c|}{ Rootstock types } & \multicolumn{5}{|c|}{ Rootstock types } & \\
\hline & 1 & 2 & 3 & 4 & M & Means & 1 & 2 & 3 & 4 & Means & \\
\hline 10. & 0.17 & 11.10 & 10.9 & 9311. & .17 & 10.84 & 10.17 & 11.10 & 10.93 & 11.17 & 10.84 & 10.84 \\
\hline 15 & 0.95 & 11.90 & 10.9 & 9311. & .37 & 11.29 & 10.43 & 11.75 & 10.85 & 12.10 & 11.28 & 11.29 \\
\hline 30 & 1.12 & 11.58 & 10.9 & \begin{tabular}{l|l}
92 & 12.
\end{tabular} & .18 & 11.45 & 10.77 & 11.33 & 10.67 & 12.23 & 11.25 & 11.35 \\
\hline 45 & 1.40 & 12.70 & 10.9 & 9312. & 2.47 & 11.88 & 10.90 & 12.23 & 11.00 & 11.82 & 11.49 & 11.68 \\
\hline 60 & 0.73 & 12.40 & 10.9 & 9012. & 2.53 & 11.64 & 10.70 & 12.33 & 11.33 & 12.93 & 11.83 & 11.73 \\
\hline 75 & 0.67 & 12.03 & 311.5 & 5012. & 2.13 & 11.58 & 10.90 & 12.33 & 10.93 & 12.67 & 11.71 & 11.65 \\
\hline 90 & 0.77 & 11.50 & 010.1 & 1311. & .97 & 11.09 & 10.80 & 12.67 & 10.67 & 12.40 & 11.63 & 11.36 \\
\hline \begin{tabular}{l|l} 
Means & 10.
\end{tabular} & 0.83 & 11.89 & 910.8 & 8911. & 1.97 & 11.40 & 10.67 & 11.96 & 10.91 & 12.19 & 11.43 & \\
\hline \multicolumn{13}{|c|}{ Second season (2006) } \\
\hline $0^{\circ}$ & 1.53 & 12.27 & 711.8 & & 11.93 & 11.88 & 11.53 & 12.27 & 11.80 & 11.93 & 11.88 & 11.88 \\
\hline 15 & 1.93 & 12.11 & 111.9 & & 12.23 & 12.04 & 12.07 & 12.30 & 12.17 & 12.37 & 12.23 & 12.13 \\
\hline 30 & 2.601 & 12.32 & 212.3 & & 12.35 & 12.40 & 12.25 & 12.17 & 11.25 & 12.28 & 11.99 & 12.19 \\
\hline 45 & 3.27 & 13.43 & 311.9 & & 12.80 & 12.86 & 11.77 & 12.37 & 11.67 & 12.47 & 12.07 & 12.46 \\
\hline 60 & 2.80 & 13.50 & 0 & & 12.88 & 12.81 & 12.47 & 13.20 & 11.72 & 13.17 & 12.64 & 12.73 \\
\hline 75 & 2.93 & 13.27 & 712.7 & & 13.30 & 13.05 & 13.10 & 13.67 & 12.70 & 13.43 & 13.23 & 13.14 \\
\hline \begin{tabular}{l|l} 
Means & 12 \\
\end{tabular} & 2.51 & 12.82 & 212.1 & & 12.58 & 12.51 & 12.20 & 12.66 & 11.88 & 12.61 & 12.34 & \\
\hline \multicolumn{6}{|c|}{ Rootstock means } & \multicolumn{6}{|c|}{ Abbreviations: } & \\
\hline Rootstock & 1 & 1 & 2 & 3 & 4 & \multicolumn{4}{|c|}{$1=$ Sour orange } & \multicolumn{3}{|l|}{$2=$ Carrizo } \\
\hline $1^{\text {st }}$ season & 10.7 & .7511 & 1.931 & 10.90 & 12.08 & \multicolumn{4}{|c|}{$3=$ Cleopatra } & \multicolumn{3}{|c|}{$4=$ Citrumelo } \\
\hline $2^{\text {nd }}$ season & 12.3 & .3512 & $2.74 \sqrt{1}$ & 12.00 & 12.60 & \multicolumn{3}{|c|}{$A=$ Storage Temp. } & \multicolumn{4}{|c|}{ B=Rootstock types } \\
\hline \multicolumn{6}{|c|}{ L.S.D. at $5 \%$} & \multicolumn{7}{|c|}{$\mathrm{C}=$ Storage period in days } \\
\hline Factors & A & A & B & 3 & & C & $a * b$ & $a^{*}$ & & $\mathrm{~b} * \mathrm{c}$ & & *b*c \\
\hline $1^{\text {st }}$ season & N.S & s. & 0.19 & & 0.25 & 252 & N.S. & 0.3 & & 0.304 & & N.S. \\
\hline $2^{\text {nd }}$ season & 0.0 & 089 & 0.12 & 26 & 0.15 & 155 & 0.178 & 0.2 & & 0.310 & & N.S. \\
\hline
\end{tabular}

These results are in line with those obtained by Ramanjulu and Reddy (1989), Schirra (1992) and Mohamed et al. (2003) who found that total soluble solids increased while total acidity and ascorbic acid decreased with prolonging of storage * period. On contrast, these results disagree with those mentioned by $S u$, et al. (1988) who reported that total soluble solids, total acidity and ascorbic acid contents of orange remained constant during storage. 
Table 8. Effect of four rootstocks and cold storage on total acidity percentage during storage of "Hernandina" Clementine fruits in 2005/2006 and 2006/2007 seasons.

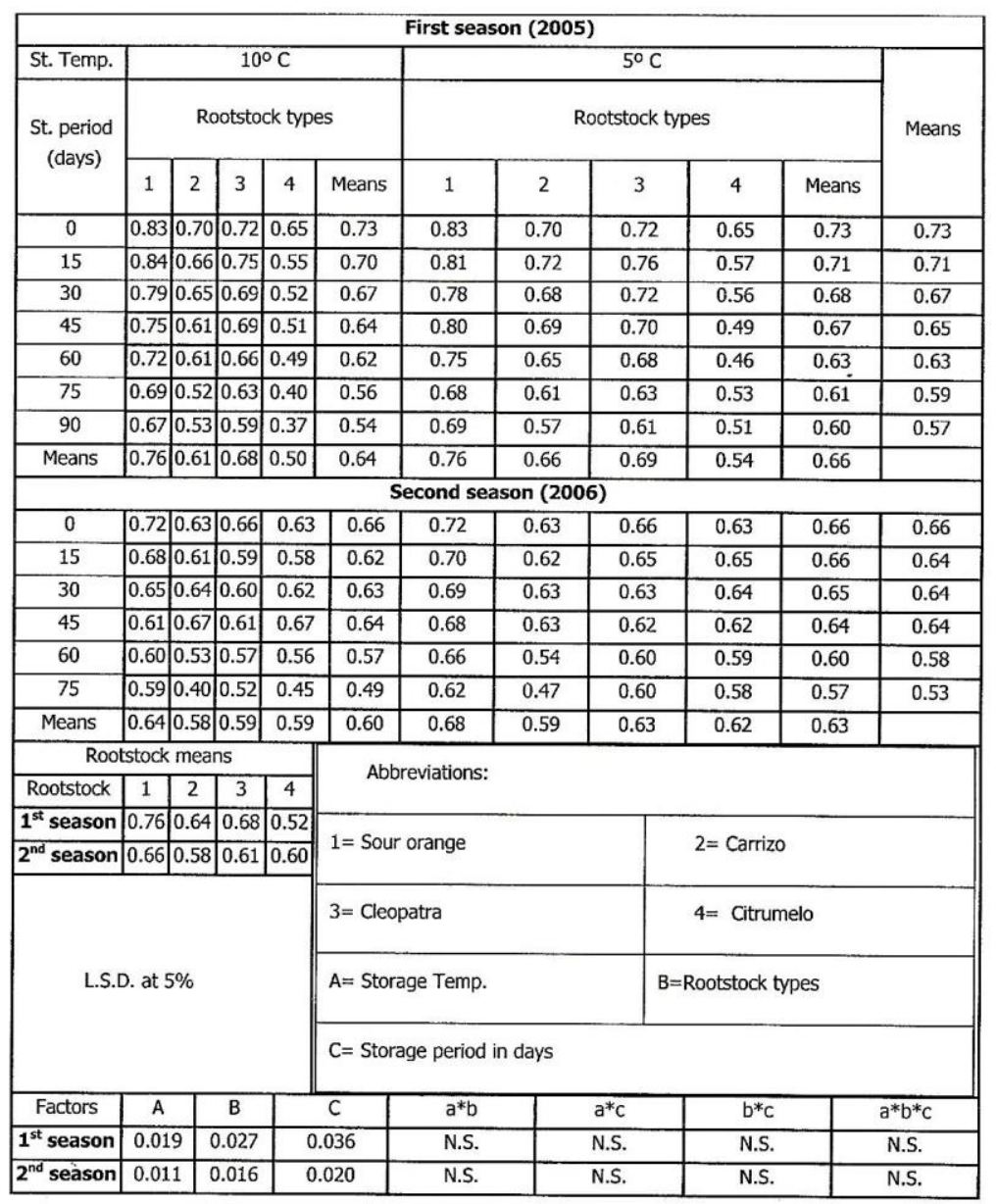

On the other side, these results agree with those obtained by D'Hallewin et al. (1994), Reynaldo (1999), Ali (2002) and El-Hilali et al. (2003) who mentioned that total soluble solids, total acidity and ascorbic acid contents were affected by rootstock types. On contrast, El-Zeftawi et al. (1989) mentioned that Valencia orange fruit was not influenced by rootstock types. 
1520 PHYSIOLOGICAL STUDIES ON THE EFFECT OF FOUR ROOTSTOCKS ON HERNANDINA CLEMENTINE FRUITS II- FRUIT STORABILITY

Table 9. Effect of four rootstocks and cold storage on TSS/TA ratio during storage of "Hernandina" Clementine fruits in 2005/2006 and 2006/2007 seasons.

\begin{tabular}{|c|c|c|c|c|c|c|c|c|c|c|c|c|}
\hline \multicolumn{13}{|c|}{ First season (2005) } \\
\hline St. Temp. & \multicolumn{5}{|c|}{$10^{\circ} \mathrm{C}$} & \multicolumn{6}{|c|}{$5^{\circ} \mathrm{C}$} & \multirow{3}{*}{ Means } \\
\hline \multirow{2}{*}{$\begin{array}{c}\text { St. period } \\
\text { (days) }\end{array}$} & \multicolumn{5}{|c|}{ Rootstock types } & \multicolumn{6}{|c|}{ Rootstock types } & \\
\hline & 1 & 2 & 3 & 4 & Means & 1 & 2 & & 3 & 4 & Means & \\
\hline 0 & 12.2 . & -15.8 & 15.2 & 17.2 & 15.12 & 12.2 & 15.8 & & 15.2 & 17.2 & 15.12 & 15.12 \\
\hline 15 & 13.0 & 18.0 & 14.6 & 20.6 & 16.54 & 12.9 & 16.3 & & 14.3 & 21.3 & 16.20 & 16.37 \\
\hline 30 & 14.0 & 17.7 & 15.8 & 23.2 & 17.69 & 13.8 & 16.8 & & 14.9 & 22.0 & 16.88 & 17.28 \\
\hline 45 & 15.2 & 20.9 & 15.8 & 24.4 & 19.06 & 13.7 & 17.8 & & 15.8 & 24.2 & 17.86 & 18.46 \\
\hline 60 & 14.9 & 20.5 & 16.5 & 25.7 & 19.39 & 14.4 & 19.0 & & 16.6 & 28.0 & 19.50 & 19.45 \\
\hline 75 & 15.4 & 23.3 & 18.2 & 30.5 & 21.86 & 15.9 & 20.4 & & 17.4 & 24.1 & 19.43 & 20.65 \\
\hline 90 & 16.0 & 21.7 & 17.0 & 32.6 & 21.82 & 15.6 & 22.2 & & 17.4 & 24.1 & 19.83 & 20.82 \\
\hline \begin{tabular}{l|l} 
Means & 1
\end{tabular} & 14.391 & 19.69 & 16.16 & 24.89 & 18.78 & 14.07 & 18.3 & & 15.94 & 23.00 & 17.83 & \\
\hline \multicolumn{13}{|c|}{ Second season (2006) } \\
\hline 0 & 16.0 & 19.6 & 18.0 & 19.1 & 18.16 & 16.0 & 19.6 & & 18.0 & 19.1 & 18.16 & 18.16 \\
\hline 15 & 17.4 & 19.8 & 20.0 & 21.1 & 19.60 & 17.1 & 20.0 & & 18.7 & 18.9 & 18.67 & 19.13 \\
\hline 30 & 19.4 & 19.3 & 20.5 & 19.8 & 19.74 & 17.7 & 19.5 & & 17.7 & 19.3 & 18.55 & 19.15 \\
\hline 45 & 21.7 & 20.2 & 19.5 & 19.1 & 20.12 & 17.3 & 19.5 & & 18.9 & 20.2 & 18.96 & 19.54 \\
\hline 60 & 21.2 & 25.4 & 21.3 & 23.0 & 22.73 & 19.0 & 24.3 & & 19.6 & 22.3 & 21.29 & 22.01 \\
\hline 75 & 21.8 & 33.5 & 24.3 & 29.4 & 27.25 & 21.2 & 28.8 & & 21.2 & 23.0 & 23.54 & 25.39 \\
\hline \begin{tabular}{l|l} 
Means & 1 \\
\end{tabular} & 19.602 & 22.952 & 20.60 & 21.92 & 21.27 & 18.06 & 21.9 & & 19.01 & 20.44 & 19.86 & \\
\hline \multicolumn{5}{|c|}{ Rootstock means } & \multirow{2}{*}{\multicolumn{8}{|c|}{ Abbreviations: }} \\
\hline Rootstock & 1 & 2 & 3 & 4 & \multirow{2}{*}{\multicolumn{4}{|c|}{$1=$ Sour orange }} & & & & \\
\hline $1^{\text {st }}$ season & 14.23 & $\begin{array}{l}319.00 \\
\end{array}$ & $\begin{array}{ll}0 & 16.05 \\
\end{array}$ & 523.94 & & & & & \multicolumn{3}{|c|}{$2=$ Carrizo } & \\
\hline $2^{\text {nd }}$ season & 18.83 & 322.44 & 419.80 & 21.18 & \multicolumn{4}{|c|}{ 3= Cleopatra } & \multicolumn{3}{|c|}{$4=$ Citrumelo } & \\
\hline \multirow{2}{*}{\multicolumn{5}{|c|}{ L.S.D. at $5 \%$}} & \multicolumn{4}{|c|}{$\mathrm{A}=$ Storage Temp. } & \multicolumn{3}{|c|}{$B=$ Rootstock types } & \\
\hline & & & & & \multicolumn{7}{|c|}{$C=$ Storage period in days } & \\
\hline Factors & A & & B & B & C & & $a * b$ & $a^{*} c$ & & $b^{*} \mathrm{c}$ & & $a * b * c$ \\
\hline $1^{\text {st }}$ season & 1.00 & & 1.4 & 41 & 1.86 & & N.S. & N.S. & & N.S. & & N.S. \\
\hline $2^{\text {nd }}$ season & 0.63 & & 0.8 & 89 & 1.09 & & N.S. & 1.54 & & 2.18 & & N.S. \\
\hline
\end{tabular}


Table 10. Effect of rootstock types and storage temperatures on Hernandina Clementine fruits V.C contents during storage.

\begin{tabular}{|c|c|c|c|c|c|c|c|c|c|c|c|}
\hline \multicolumn{12}{|c|}{ First season (2005) } \\
\hline St. Temp. & \multicolumn{5}{|c|}{$10^{\circ} \mathrm{C}$} & \multicolumn{5}{|c|}{$5^{\circ} \mathrm{C}$} & \multirow{3}{*}{ Means } \\
\hline \multirow{2}{*}{$\begin{array}{c}\text { St. period } \\
\text { (days) }\end{array}$} & \multicolumn{5}{|c|}{ Rootstock types } & \multicolumn{5}{|c|}{ Rootstock types } & \\
\hline & 1 & 2 & 3 & 4 & $\mid \begin{array}{c}\text { Mean } \\
s\end{array}$ & 1 & 2 & 3 & 4 & Means & \\
\hline 0 & 19.5 & 19.8 & 17.1 & 19.3 & 18.91 & 19.5 & 19.8 & 17.1 & 19.3 & 18.91 & 18.91 \\
\hline 15 & 17.7 & 19.1 & 16.2 & 18.4 & 17.85 & 18.0 & 18.8 & 16.8 & 17.4 & 17.75 & 17.80 \\
\hline 30 & 16.8 & 18.5 & 15.3 & 17.4 & 17.00 & 17.1 & 18.5 & 16.1 & 16.8 & 17.14 & 17.07 \\
\hline 45 & 15.9 & 17.1 & 15.1 & 16.4 & 16.14 & 16.5 & 17.8 & 14.6 & 15.4 & 16.08 & 16.11 \\
\hline 60 & 14.1 & 14.5 & 13.0 & 13.6 & 13.80 & 14.8 & 17.2 & 12.5 & 14.3 & 14.70 & 14.25 \\
\hline 75 & 11.7 & 11.2 & 9.4 & 8.0 & 10.08 & 9.3 & 10.9 & 8.2 & 11.1 & 9.86 & 9.97 \\
\hline 90 & 7.9 & 9.5 & 7.6 & 8.4 & 8.33 & 7.7 & 9.8 & 8.7 & 11.0 & 9.29 & 8.81 \\
\hline Means & $\begin{array}{c}14.8 \\
1\end{array}$ & $\begin{array}{c}15.6 \\
6\end{array}$ & $\begin{array}{c}13.3 \\
9\end{array}$ & $\begin{array}{c}14.4 \\
9\end{array}$ & 14.59 & 14.71 & 16.10 & 13.43 & 15.04 & 14.82 & \\
\hline \multicolumn{12}{|c|}{ Second season (2006) } \\
\hline 0 & 21.6 & 24.0 & 23.3 & 21.7 & 22.64 & 21.6 & 24.0 & 23.3 & 21.7 & 22.64 & 22.64 \\
\hline 15 & 19.7 & 21.0 & 18.3 & 17.9 & 19.21 & 20.2 & 21.3 & 19.1 & 18.1 & 19.69 & 19.45 \\
\hline 30 & 17.7 & 18.3 & 14.0 & 14.1 & 16.03 & 18.9 & 18.7 & 14.9 & 14.5 & 16.74 & 16.39 \\
\hline 45 & 16.3 & 15.4 & 13.5 & 12.3 & 14.38 & 16.9 & 16.6 & 13.9 & 12.5 & 14.96 & 14.67 \\
\hline 60 & 15.3 & 13.5 & 13.0 & 9.2 & 12.75 & 16.4 & 14.0 & 11.5 & 9.2 & 12.76 & 12.76 \\
\hline 75 & 11.7 & 10.1 & 8.5 & 7.1 & 9.35 & 13.2 & 11.6 & 10.2 & 8.1 & 10.78 & 10.07 \\
\hline Means & $\begin{array}{c}17.0 \\
3\end{array}$ & $\begin{array}{c}17.0 \\
4\end{array}$ & $\begin{array}{c}15.1 \\
2\end{array}$ & $\begin{array}{c}13.7 \\
3\end{array}$ & 15.73 & 17.86 & 17.70 & 15.49 & 14.00 & 16.26 & \\
\hline \multicolumn{6}{|c|}{ Rootstock means } & \multirow{2}{*}{\multicolumn{6}{|c|}{ Abbreviations: }} \\
\hline Rootstock & & 1 & 2 & 3 & 4 & & & & \multirow{2}{*}{\multicolumn{3}{|c|}{$2=$ Carrizo }} \\
\hline \multirow{2}{*}{$1^{\text {st }}$ season } & \multirow{2}{*}{\multicolumn{2}{|c|}{14.76}} & \multirow{2}{*}{15.88} & \multirow{2}{*}{13.41} & \multirow{2}{*}{\begin{tabular}{|c||}
14.7 \\
7 \\
\end{tabular}} & \multicolumn{3}{|c|}{$1=$ Sour orange } & & & \\
\hline & & & & & & \multicolumn{3}{|c|}{ 3= Cleopatra } & \multicolumn{3}{|c|}{$4=$ Citrumelo } \\
\hline $2^{\text {nd }}$ season & \multicolumn{2}{|c|}{17.45} & 17.37 & 15.31 & \multirow[t]{2}{*}{$\begin{array}{c}13.8 \\
6\end{array}$} & \multicolumn{3}{|c|}{$\mathrm{A}=$ Storage Temp. } & \multicolumn{3}{|c|}{$B=$ Rootstock types } \\
\hline \multicolumn{5}{|c|}{ L.S.D. at $5 \%$} & & \multicolumn{6}{|c|}{$\mathrm{C}=\mathrm{Storage}$ period in days } \\
\hline Factors & & A & & B & 3 & & C & $a * b$ & $a * c$ & $\mathrm{~b}^{*} \mathrm{c}$ & $a * b * c$ \\
\hline $\begin{array}{l}1^{\text {st }} \\
\text { season }\end{array}$ & & 0.43 & & N.S & S. & & 80 & N.S. & N.S. & N.S. & N.S. \\
\hline $\begin{array}{l}2^{\text {nd }} \\
\text { season }\end{array}$ & & .28 & & 0.39 & 39 & & 48 & N.S. & 0.67 & 0.95 & N.S. \\
\hline
\end{tabular}




\section{REFERNCES}

1. Alferez, F., J. M. Sala, M. T. S. Ballesta, M. Mulas, M. T. Lafuente and L. Zacarias. 2005. A comparative study of the post-harvest performance of an ABAdeficient mutant of oranges. I. Physiological and quality aspects. Post-harvest Biology and Technology, 37(3): 222-231.

2. Ali, G. M., 2002. Effect of four citrus rootstocks on fruit quality and storability of Fremont tangerine the second Inter. Conf. Hort. Sci, 10-12 sep 2002, Kafr ElSheikh, Tanta Univ., Egypt, 312-326.

3. Association of Official Analytical Chemists. 1995. Official methods of analysis, $14^{\text {th }}$ ed. Benjamin Franklin Station. Washington D.C. 20044, U.S.A. Pp. 490-510

4. D’Hallewin, D. G., D. Mura, A. Piga, M. Pala, G. Lovicu and P. Sass.1994. Rootstock effects on post-harvest physiological and pathological behavior of Avana mandarin. International symposium on post-harvest treatment of horticultural crops, Kecskemet, Hungry, 30 Aug-3Sep. 1993. Acta- Hortculturae, No.368, 395-402.

5. El-Hilali, F., A. Remah, A. Ait-Oubahou, O. Akhayat. 2003. Effect of rootstock on fruit quality and peel peroxidase activity of 'Afourer' mandarin fruit at low temperature storage. Journal of Food, Agriculture and Environment, 1(2): 234 237. (Cab Abst., Computer Search).

6. El-Zeftawi B.M., I.D. Peggie and D. C. Minns. 1989. Post-harvest treatments, storage temperature, and rootstocks in relation to storage disorders and fruit 'quality of Valincia oranges. J. Hort. Sci, 64 (3): 373-378.

7. Farih, A., E. B. Nadori, H. Bellouch, M. Ouammou, F. Abdelali, A. Jrifi and H. Boukhriss. 1995. Research on the storability of Clementine fruits: effect of clone and rootstock on the development of rots in cold storage. Al-Awamia., (88): 57 66. (Cab Abstracts Computer Search).

8. Mc Guire, R. G. 1992. Reporting of objective colour measurements. HortScience, Vol., 27(12): 1254-1255.

9. Mohamed, M. A. A., A. A. Abd El-Hafeez and S. M. A. Mehaisen. 2002. Response of Valencia orange and Marsh seedless grapefruit to post-harvest hot water dips and fruits storage temperature. Annals of Agric. Sc., Moshtohor, Zagazig Univ., 40(4): 2247-2264.

10. Mohamed, M. A. A., A. A. Abd El-Hafeez and S. M. A. Mehaisen. 2003. Effect of post-harvest treatments with some safe compounds on fruit properties of 
Valencia orange and Marsh seedless grapefruit fruits during storage. Annals of Agric. Sc., Moshtohor, Zagazig Univ., 41(3): 1223-1237.

11. Ramanjulu, V.V. and M.R.S. Reddy. 1989. Post- Infection changes in Sathgudi sweet orange fruits caused by Gloeosporium limetticola. Indian Phytopathology, 42 (1): 191- 192.

12. Reynaido, I.M. 1999. The influence of rootstock on the post-harvest behavior of Ruby Red grapefruit. Cultivos-Tropicales., 20(2): 37-40. (Cab Abstracts Computer Search).

13. Ritenour, M. A., H. T. Dou, K. D. Bowman, B. J. Boman, E. Stover and W. S. Castle. 2004. Effect of rootstock on stem-end rind breakdown and decay of fresh citrus. Hort. Technology, 14(3): 315-319. (Cab Abstracts Computer Search).

14. Schirra, M. 1992. Behavior of star Ruby grapefruit under chilling storage temperature. Post-harvest Biology and Technology. 2, 315-327.

15. Schirra, M. Agabblo, G. D'Hallewin, M. pala. and R. Ruggiu. 1997. Response of Tarocco oranges to picking data, post harvest hot water dips, and chilling storage temperature. Journal of Agricultural and Food Chemistry, 45 (8): 3216 3220.

16. Snedecor, G. W. and W. G. Cochran. 1980. Statistical methods. Oxford and J.B.H. Publishing Com. 7th Edition.

17. Su, M., X., Z. Y. Wang, M. Q. Li and Li P. W. 1988. Effect of postharvest treatments on control of brown blotch of orange fruits. Acta Phyto Physiological Sinca, 14(4):318-322.

18. Valbuena, M., 1996. Agronomic performance of some citrus stock-scion combinations in middle zone of the river Guasare, Zulia, Venezuela. Revista de la Facultad de Agronomía Universidad del Zulia, 11:1,1-12:1map. 
1524 PHYSIOLOGICAL STUDIES ON THE EFFECT OF FOUR ROOTSTOCKS ON HERNANDINA CLEMENTINE FRUITS II- FRUIT STORABILITY.

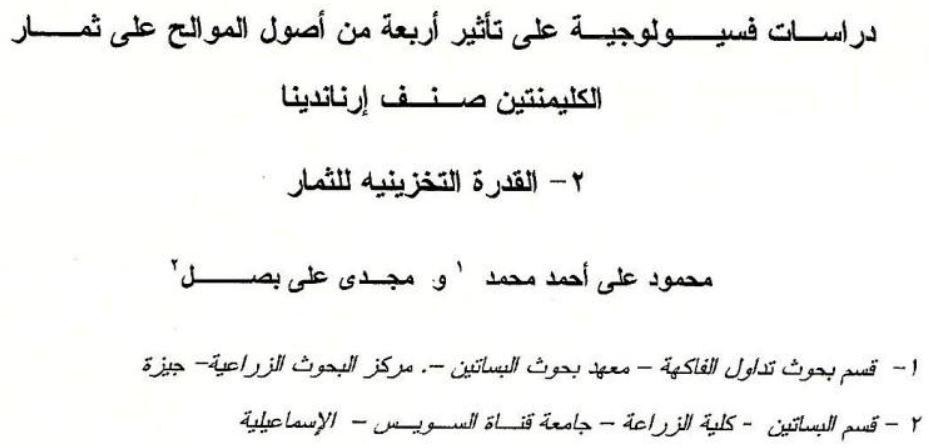

تعتبر الموالح من المحاصبل التصديرية الهامة بالنسبة للذخل التومي المصري ــ وقد بلغت

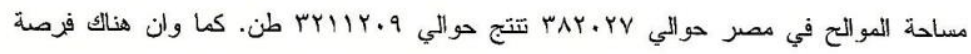
كبيرة لزيادة المصدر من الموالح غير الثقليدية إلى السوق الأوروبي. أجريت هذه الدراسة بمعهد بحوث البساتين - قسم بحوث تداول الفاكهة خلال موسمي ه... ب و

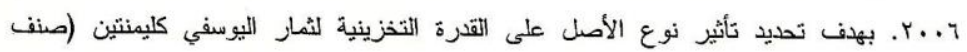

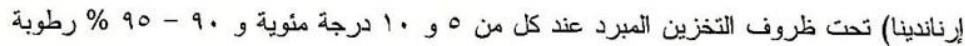

جمعت الثمار من أشجار عمر V سنوات منزرعة بمزرعة خاصة بمنطقة وادي الملاك منزرعة فى ارض رمليه ومطعومة على أربعة أصول هي النارنج - اليوسفي كليوباترا - الكاريزو سترانج السوينجل ستروميلو عند وصون الثمار إتي مرحلة اكتمال النمو (الدراسة الأولى من البحث) ونقلت

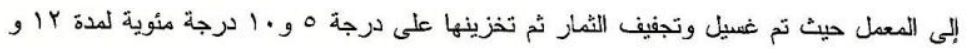
• 1 أسابيع في الموسم الأول والثاني على الترتيب. ثُم فحص الثمار دوريا كل خمسة عشر يوما للدراسة التغيرات في الخواص الطبيعية و الكيميائية للثمار أثثاء التخزين.

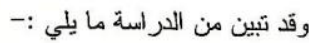
زادت نسبة التالف والفقد في الوزن في الثمار المخزنة مع نقدم الثمار في التخزين وبغض النظر عن درجة حرارة التخزين. كذلك زادت نسبة المواد الصلبة الذائبة وكذلك نسبة المواد الصلبة الذائبة إلى نسبة الحموضة الكلية بينما انذفض محتوى الثمار من العصير وكنلك صلابة الثمار و الأحماض

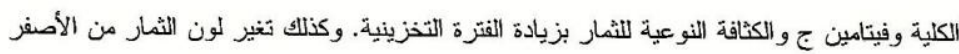
إلى الأصفر البرتثالي ثم إلى البرتقالي ثم إلى البرثقالي المحمر مع ثقدم الثمار في التخزين. 
كانت نسبة الفقد في الوزن وكذلك نسبة المواد الصلبة الذائبة و نسبة المواد الصلبة الذائبة إلى نسبة الحموضة الكلية في ثمار اليوسفي كليمنتين المحمولة على أصل النارنج القل منها هقارنة

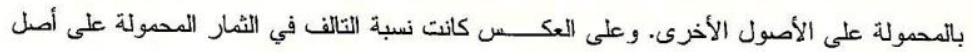
النارنج وكذلك الكثافة النوعية و الصلابة و ونسبة الحموضة الكلية أعلى منها بالمقارنة بالثمار

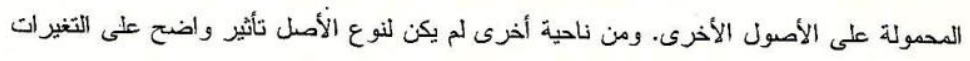

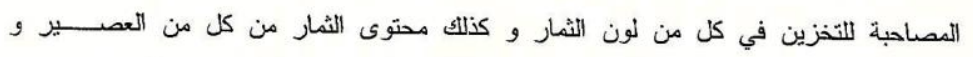

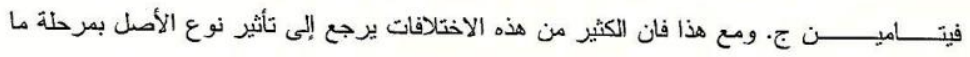
قبل الحصاد وليس أثناء التخزين. أدى التخزين على درجة ـ ا مئوية إلى زيادة نسبة التالف و الفقد في الوزن وكذلك الإسر اع من عملية التلوين وزيادة نسبة المواد الصلبة الذائبة ونسبة المواد الصلبة الذائبة إلى الحموضة الكلية ونقص محتوى الثمار من العصير والحموضة الكلية وفيتامين ج وزيادة النقص في الكثافة النوعية

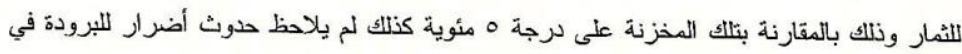
تلاك المخزنة على درجة م مئوية كما لم يؤدى إلى زيادة نسبة الثالف في الثمار المخزنة. وعليه يمكن القول أن ثمار اليوسفي كليمنتين صنف إرناندينا يمكن تخزينها على درجة ه مئوية

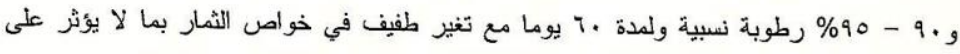
خصائص الجودة في الثمار بييما كان تأثير نوع الأصل (الأصول تحت الدراسة) على القدرة التخزينية للثمارمحــــــودا. 